\title{
Codelivery of thioridazine and doxorubicin using nanoparticles for effective breast cancer therapy
}

This article was published in the following Dove Press journal:

International Journal of Nanomedicine

8 September 2016

Number of times this article has been viewed

\author{
Xun Jin ${ }^{1, *}$ \\ Bingwen Zou ${ }^{2}, *$ \\ Li Luo',* \\ Chuanhong Zhong ${ }^{3}$ \\ Peilan Zhang' \\ Hao Cheng' \\ Yanfang Guo' \\ Maling Gou'
}

'State Key Laboratory of Biotherapy and Cancer Center, West China Hospital, Sichuan University, and Collaborative Innovation Center for Biotherapy, 2Department of Thoracic Oncology, Cancer Center, West China Hospital, Medical School, Sichuan University, Chengdu, ${ }^{3}$ Department of Neurosurgery, The Affiliated Hospital of Luzhou Medical College, Luzhou, Sichuan Province, People's Republic of China

*These authors contributed equally to this work
Correspondence: Maling Gou State Key Laboratory of Biotherapy and Cancer Center, West China Hospital, Collaborative Innovation Center for Biotherapy, Sichuan University, Chengdu 61004I, People's Republic of China Tel +862885I6 406I

$\mathrm{Fax}+862885164060$

Email goumaling@scu.edu.cn

\begin{abstract}
Cancer chemotherapy can benefit from the combination of different anticancer drugs. Here, we prepared doxorubicin (Dox)- and thioridazine (Thio)-coloaded methoxy poly(ethylene glycol)-poly(L-lactic acid) (MPEG-PLA) nanoparticles (NPs) for breast cancer therapy. These NPs have an average particle size of $27 \mathrm{~nm}$. The drug loading efficiencies of Thio and Dox are $4.71 \%$ and $1.98 \%$, respectively. Compared to the treatment of Thio or Dox alone, the combination of Thio and Dox exhibited a synergistic effect in inhibiting the growth of 4T1 breast cancer cells in vitro. In addition, the Thio- and Dox-coloaded MPEG-PLA NPs could efficiently suppress the growth of breast cancer cells in vivo. This study suggests that Thio- and Dox-coloaded MPEG-PLA NPs might have potential applications in breast cancer treatment.
\end{abstract}

Keywords: thioridazine, doxorubicin, codelivery, nanoparticles, breast cancer

\section{Introduction}

Breast cancer is one of the most common tumors in women worldwide and remains the leading cause of cancer-related death among females in less developed countries. ${ }^{1,2}$ Although much progress has been made in breast cancer therapeutics, $\sim 30 \%$ of breast cancer patients treated in early stage suffer from recurrence due to therapeutic resistance, such as chemoresistance. ${ }^{3}$ Hence, it is critical to develop effective strategies to overcome drug resistance for breast cancer therapy.

Instead of single drug treatment, simultaneous administration of multiple drugs is an attractive way to maximize the therapeutic response of chemotherapy and minimize the occurrence of multidrug resistance. ${ }^{4,5}$ Thioridazine (Thio), a phenothiazine derivative, is a piperidine antipsychotic drug that was recently found to inhibit cancer cell growth. ${ }^{6-8}$ Doxorubicin (Dox), an anthracycline antibiotic, is one of the most widely applied anticancer drugs for various malignancies, including breast, prostate, and colon cancers. ${ }^{9-12}$ However, serious side effects, such as the dose-dependent cardiotoxicity, myelosuppression, and nephrotoxicity, markedly limit its clinical use. ${ }^{13,14}$ The strategy of combining two chemotherapeutic agents has been proved to be able to achieve the synergetic anticancer effects. Lv et a ${ }^{15}$ used the combination of Dox and paclitaxel to treat lung cancer. Zhang et al ${ }^{16}$ combined Dox and dasatinib for breast cancer. In this study, the combination of Thio and Dox was designed to maximize the anticancer activity and minimize drug resistance.

Over the past decades, nanotechnology has made great contribution to the development of drug delivery systems. ${ }^{17-19}$ Encapsulation of anticancer drugs by polymeric nanoparticles (NPs) facilitates drug distribution in tumor tissues through an enhanced permeability and retention (EPR) effect that shows better pharmacokinetics profiles in vivo and reduces multidrug resistance of malignances, leading to enhanced anticancer effects. ${ }^{20-23}$ Various micelles, such as MPEG poly( $\varepsilon$-caprolactone and methoxy 
poly(ethylene glycol)-poly(L-lactic acid) (MPEG-PLA), have been extensively used as effective drug delivery systems. ${ }^{24}$ MPEG-PLA, an amphiphilic polymer, is appropriate for drug delivery due to its biodegradation ability and low clearance rate. Here, we coencapsulated Dox and Thio with MPEGPLA polymer in two steps, forming Thio-Dox-coencapsulated NPs. The prepared NPs were characterized in size distribution, morphology, and in vitro drug release behavior. The anticancer effect against breast cancer 4T1 cells was also evaluated both in vitro and in vivo. Our results suggest that the codelivery of Dox and Thio using an MPEG-PLA nanocarrier has a potential application in breast cancer chemotherapy.

\section{Materials and methods}

\section{Materials}

MPEG-PLA (MPEG:PLA molar ratio =50:50, molecular weight $=4,000 \mathrm{~g} / \mathrm{mol}$ ) was purchased from DaiGang (Jinan, Shandong, People's Republic of China). Dox hydrochloride was provided by Melonepharma Co Ltd (Dalian, Liaoning, People's Republic of China). Thio was supplied by the National Institute for the Control of Pharmaceutical and Biological Products (Beijing, People's Republic of China). Methanol and acetonitrile were purchased from Kermel (Tianjin, People's Republic of China). All other chemicals and solvents were obtained from Kelong Chemical Reagent Factory (Chengdu, Sichuan, People's Republic of China), and they were at least of analytical grade.

Mice breast cancer cell line 4T1, mice colon cancer cell line C26, and human embryo kidney cell line HEK293 were purchased from American Type Culture Collection (ATCC, Manassas, VA, USA). The Ethical Committee of the West China Hospital, Sichuan University, approved this study. RPMI1640 complete medium, fetal bovine serum (FBS), penicillin, and streptomycin were purchased from HyClone (Logan, UT, USA). All cells were maintained in RPMI1640 complete medium supplemented with $10 \%$ (FBS), $100 \mathrm{U} / \mathrm{mL}$ penicillin, and $100 \mu \mathrm{g} / \mathrm{mL}$ streptomycin at $37^{\circ} \mathrm{C}$ in $5 \% \mathrm{CO}_{2}$ atmosphere. All mice were obtained from the Beijing HFK Bioscience (Beijing, People's Republic of China). Mice were humanely treated according to the guidelines of the Institutional Animal Care and Treatment Committee of Sichuan University, and all animal procedures were approved and controlled by the Institutional Animal Care and Treatment Committee of Sichuan University.

\section{Preparation of Dox, Thio, and Thio- Dox-loaded NPs}

\section{Preparation of Thio NPs}

To obtain the Thio/MPEG-PLA NPs, $95 \mathrm{mg}$ MPEG-PLA and $5 \mathrm{mg}$ Thio were codissolved in $2 \mathrm{~mL}$ dichloromethane, followed by evaporation under reduced pressure in a rotary evaporator at $60^{\circ} \mathrm{C}$. Subsequently, the film was rehydrated in $500 \mu \mathrm{L}$ phosphate-buffered saline (PBS; $\mathrm{pH}=7.4$ ), allowing the self-assembly of Thio/MPEG-PLA NPs.

\section{Preparation of codelivery Thio-Dox NPs}

To prepare the Thio-Dox NPs, $2.5 \mathrm{mg}$ Dox aqueous solution was added into the Thio/MPEG-PLA solution dropwise under moderate stirring. After 20 minutes, the Thio-Dox-loaded NPs were obtained and stored at $4{ }^{\circ} \mathrm{C}$ for future use.

Drug loading (DL) and encapsulation efficiency (EE) of codelivery Thio-Dox NPs were calculated from the following formulas:

$$
\begin{aligned}
& \text { Drug loading (DL, } \% \mathrm{w} / \mathrm{w}) \\
& =\frac{\text { Weight of drug in nanoparticles }}{\text { Weight of nanoparticles }} \times 100 \%
\end{aligned}
$$

$$
\begin{aligned}
& \text { Encapsulation efficiency (EE, } \% \mathrm{w} / \mathrm{w}) \\
& =\frac{\text { Weight of drug in nanoparticles }}{\text { Weight of total drug used }} \times 100 \%
\end{aligned}
$$

\section{Characterization of codelivery Thio-Dox NPs}

Particle size analysis

The particle size of prepared NPs was determined by Malvern Nano ZS90 (Malvern Instruments, Malvern, UK). The temperature was kept at $25^{\circ} \mathrm{C}$ during the measuring process. All results were the mean of three test runs.

\section{Morphology study}

The morphology of the prepared NPs was observed under a transmission electron microscope (H-6009IV; Hitachi Ltd., Tokyo, Japan). NPs were diluted with distilled water and placed on a copper grid covered with nitrocellulose. Samples were negatively stained with phosphotungstic acid and dried at room temperature.

\section{In vitro drug release study}

In vitro drug release behaviors of free Thio, free Dox, and Thio-Dox-loaded NPs were determined by dialysis method. ${ }^{24}$ Briefly, $2 \mathrm{~mL}$ of free Thio, or free Dox solution, or Thio-Doxloaded NPs were placed in dialysis bags (molecular weight cutoff, $3.5 \mathrm{kDa}$ ). The dialysis bags were incubated in $20 \mathrm{~mL}$ of PBS (prewarmed to $37^{\circ} \mathrm{C}$ and $\mathrm{pH}=7.4$ and $\mathrm{pH}=5.5$ ) or $20 \mathrm{~mL}$ of PBS (prewarmed to $37^{\circ} \mathrm{C}$ and $\mathrm{pH} 7.4$ ) containing $10 \% \mathrm{FBS}$ at $37^{\circ} \mathrm{C}$ with shaking at $100 \mathrm{rpm}$ in a $50 \mathrm{~mL}$ tube. At predetermined time points, $2 \mathrm{~mL}$ of release media was collected, and the incubation mediums were replaced with fresh 
incubation mediums. The amount of released drugs was quantified by high-performance liquid chromatography (HPLC, Waters 2695; Waters Corporation, Milford, MA, USA). For Dox, chromatography was performed on a Kromasil C18 column $\left(4.6 \times 250 \mathrm{~mm}^{2}, 5 \mu \mathrm{m}\right.$ for Dox $)$. The effluents were monitored at excitation wavelength of $233 \mathrm{~nm}$ at $40^{\circ} \mathrm{C}$. The mobile phase consisted of a gradient of methanol, dipotassium phosphate $(0.01 \mathrm{~mol} / \mathrm{L})$, and acetonitrile $(8: 9: 3, \mathrm{pH}=3.52)$ with a flow rate of $1 \mathrm{~mL} / \mathrm{min}$. For Thio, chromatography was performed on a Kromasil C18 column $\left(4.6 \times 150 \mathrm{~mm}^{2}, 5 \mu \mathrm{m}\right)$. The effluents were monitored at excitation wavelength of $263 \mathrm{~nm}$. The mobile phase consisted of methanol and water (70:30) with $0.3 \%$ tetramethylethylenediamine $(\mathrm{pH}=6.0)$ with a flow rate of $1 \mathrm{~mL} / \mathrm{min}$. This experiment was repeated three times, and the results were shown as mean value \pm standard deviation (SD).

\section{In vitro anticancer activity Colony formation analysis}

The growth inhibition effect of Thio in breast cancer cells was examined by the colony formation analysis. Briefly, 4T1 cells harvested at $\sim 80 \%$ confluence were suspended in RPMI1640 with 10\% FBS (100 cells/well). Then, normal saline (NS) and different concentrations of Thio $(1 \mu \mathrm{g} / \mathrm{mL}$, $2 \mu \mathrm{g} / \mathrm{mL}$, and $4 \mu \mathrm{g} / \mathrm{mL}$ ) were added into the culture medium. After incubation for 3 weeks, cell colonies were stained with gimesa. The results were shown as mean value $\pm \mathrm{SD}$.

\section{MTT assay}

The cytotoxicity of Thio and Dox was determined against C26, 4T1, and HEK293 cell lines using MTT assay. Dox and Thio used in the cytotoxicity assays were prepared in normal saline. C26, 4T1, and HEK293 cells were resuspended in complete medium and seeded in 96-well tissue culture plates at a concentration of 3,000 cells/well. The cells were allowed to attach to the surface for 12 hours and then exposed to $100 \mu \mathrm{L}$ of diluted formulations ranging from $0 \mu \mathrm{g} / \mathrm{mL}$ to $16 \mu \mathrm{g} / \mathrm{mL}$ Thio or from $0 \mu \mathrm{g} / \mathrm{mL}$ to $8 \mu \mathrm{g} / \mathrm{mL}$ Dox, compared with Thio- or Dox-loaded MPEG-PLA. After 3 days of incubation, $20 \mu \mathrm{L}$ of MTT solution $(5 \mathrm{mg} / \mathrm{mL}$ ) was added to each well, and plates were continuously incubated for an additional 4 hours. The formazan crystals produced were solubilized by adding $100 \mu \mathrm{L}$ dimethyl sulfoxide. Optical densities were read at $570 \mathrm{~nm}$ using a microplate reader. The cells incubated in culture medium alone served as a control for cell viability. Mean optical density values were used to estimate the cell viability.

\section{Synergistic effect of Thio and Dox}

A MTT method was used to evaluate the synergistic effects of Thio and Dox. Briefly, the half maximal inhibitory concentration (IC50) of Thio and Dox to breast cancer cell line 4T1 was tested with MTT. Then, a theoretical IC50 line combined with Thio and Dox was drawn up with the IC50 values of Thio $(3.5,0)$ and Dox $(0,0.9)$. To verify the synergistic effects of Thio and Dox, Thio $(0.5 \mu \mathrm{g} / \mathrm{mL}, 1 \mu \mathrm{g} / \mathrm{mL}$, $1.5 \mu \mathrm{g} / \mathrm{mL}, 2 \mu \mathrm{g} / \mathrm{mL}$, and $2.5 \mu \mathrm{g} / \mathrm{mL}$ ) was added into the cultured 4T1 cells in 96 well plates. The IC50 of Dox to 4T1 was evaluated with MTT. The IC50 of Dox to 4T1 in Thio medium was compared with the corresponding value in the straight line, and we found that a lower IC50 value of Dox, combined with Thio, to 4T1 cells compared with the theoretical value calculated via the IC50 line. Consistent results were observed in the Thio IC50 test.

\section{In vivo anticancer activity}

To evaluate the anticancer effects of Thio-Dox NPs, the mice subcutaneously transplanted breast cancer model was established. $4 \mathrm{~T} 1$ breast cancer cells $\left(5 \times 10^{5}\right.$ cells/mouse $)$ were suspended in $100 \mu \mathrm{L}$ of RPMI 1640 basic medium and subcutaneously injected into the right flank of female BALB/c mice. On post-injective day 5 , mice were randomly divided into five groups (six mice per group). At post-injective times (days 9, 12, 15, and 18), NS, Dox (5 mg/kg), Thio (10 mg/kg), Thio-Dox $(5 / 10 \mathrm{mg} / \mathrm{kg})$, and Thio-Dox NPs $(5 / 10 \mathrm{mg} / \mathrm{kg})$ formulations were given by intravenous injection. At the predetermined time points, mice were weighed, and tumor size was measured. The tumor volume was calculated with the following formula: tumor volume $=$ length $\times$ width $^{2} / 2$. The mice were sacrificed at day 23. Tumors and vital organs were obtained. Tumor weight was recorded, and vital organs were stored for further pathological section analysis.

\section{Statistical analysis}

All experimental data were presented as the mean value \pm SD. One-way analysis of variance was applied using SPSS software. $P$-values $<0.05$ were considered to be statistically significant.

\section{Results and discussion Antitumor effect of Thio in breast cancer}

Recently, it was reported that Thio could selectively target leukemic cancer stem cells, but it had no effect on normal blood stem cells. ${ }^{6}$ In this study, we examined the effect of Thio on the growth inhibition of breast cancer 4T1 cells by colony formation. As expected, Thio could suppress colony formation in a dose-dependent manner as shown in Figure $1 \mathrm{~A}$ and B. Using the concentration of $4 \mu \mathrm{g} / \mathrm{mL}$ of Thio, the colony formation inhibiting rate was $\sim 100 \%$, indicating that Thio was capable of inhibiting 4T1 cell 
A
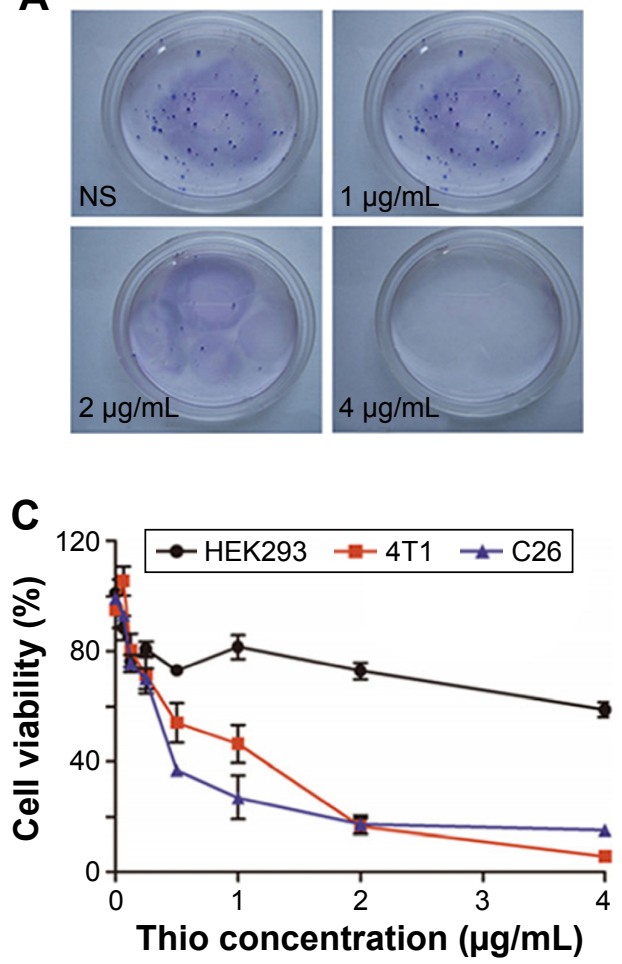

B

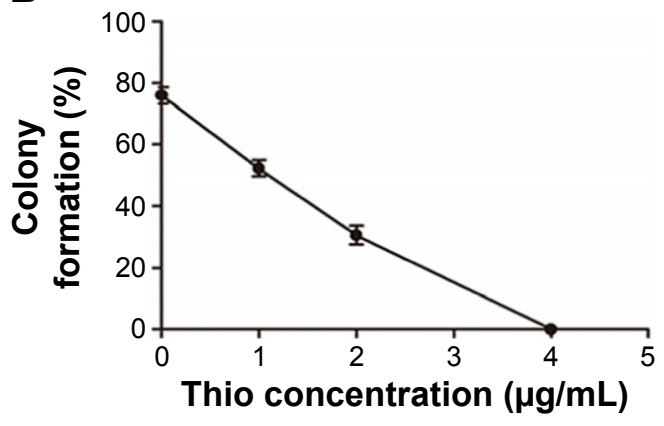

D

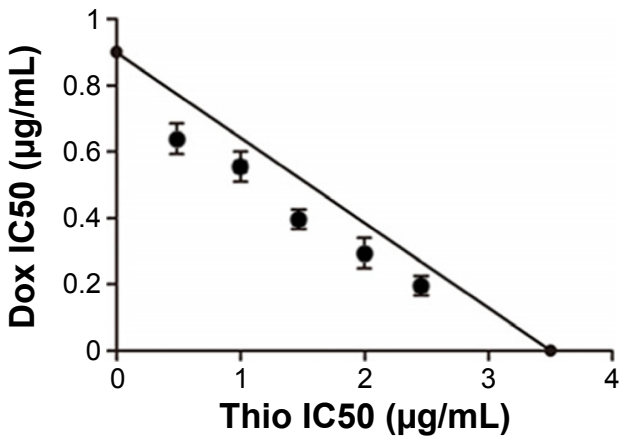

Figure I Anticancer effects of Thio in breast cancer.

Notes: (A) Colony formation of 4TI cells with Thio treatment. (B) Colony formation rate of 4TI cells using different concentrations of Thio. (C) Cytotoxicity of Thio on 4TI, C26, and HEK293 cell lines. (D) Cytotoxicity of combination of Thio and Dox on 4TI cells.

Abbreviations: Thio, thioridazine; Dox, doxorubicin; IC50, half maximal inhibitory concentration; NS, normal saline.

growth. The cytotoxicity of Thio in several other cell lines is also shown in Figure 1C. Both 4T1 breast cancer cells and $\mathrm{C} 26$ colon cancer cells, but not human embryo kidney cells HEK293, were sensitive to Thio, indicating that Thio selectively killed tumor cells while had no obvious cytotoxicity in normal cells. The IC50 value of Thio in $4 \mathrm{~T} 1$ and $\mathrm{C} 26$ cells was $0.35 \mu \mathrm{g} / \mathrm{mL}$ and $0.65 \mu \mathrm{g} / \mathrm{mL}$, respectively. We further investigated the synergistic effect of Thio and Dox by MTT analysis. As shown in Figure 1D, the IC50 value of Thio using the IC50 of Dox concentration was below the theoretical value, indicating that Thio and Dox synergistically inhibited breast cancer cell growth.

\section{Encapsulation of Thio to MPEG-PLA NPs}

NP carriers enable a better accumulation of drugs in tumor tissues through an EPR effect ability to overcome multidrug resistance with better pharmacokinetics profiles in vivo. MPEG-PLA NPs have been used to deliver several anticancer drugs due to their stability and high EE. The biodegradability of MPEG-PLA ensures the safety, and its low clearance rate would contribute to the long circulation of drugs in vivo. Therefore, we designed the Thio MPEG-PLA NPs for breast cancer treatment. The feasibility of Thio loaded to
MPEG-PLA NPs was determined by size and transmission electron microscopy analysis. As revealed in Figure 2A and $\mathrm{B}$, the average size of Thio/MPEG-PLA NPs was $25.0 \mathrm{~nm}$. To evaluate the anticancer effects of Thio-loaded MPEG-PLA NPs compared with free Thio, MTT analysis of free Thio and Thio-loaded MPEG-PLA NPs to 4T1 cells (1 day) was performed. As shown in Figure 2C, the IC50 of free Thio and Thio/MPEG-PLA NPs was $2.9 \mu \mathrm{g} / \mathrm{mL}$ and $1.8 \mu \mathrm{g} / \mathrm{mL}$, respectively. Therefore, the cytotoxicity of Thio was enhanced after encapsulation in vitro.

\section{Characterization of Thio-Dox-loaded MPEG-PLA NPs}

We further investigated whether Thio and Dox can be loaded on MPEG-PLA NPs. Successful combination of different chemotherapy drugs is usually based on specific properties of the prepared drug delivery system, such as successful incorporation, and sustained release. In recent years, attempts have been made to deliver chemotherapy drugs simultaneously through various delivery systems, such as micelles, liposomes, microcapsule, and inorganic NPs. ${ }^{25}$ For example, codelivery of Dox and curcumin in poly(D,Llactide-co-glycolide) NPs has been demonstrated to be an 

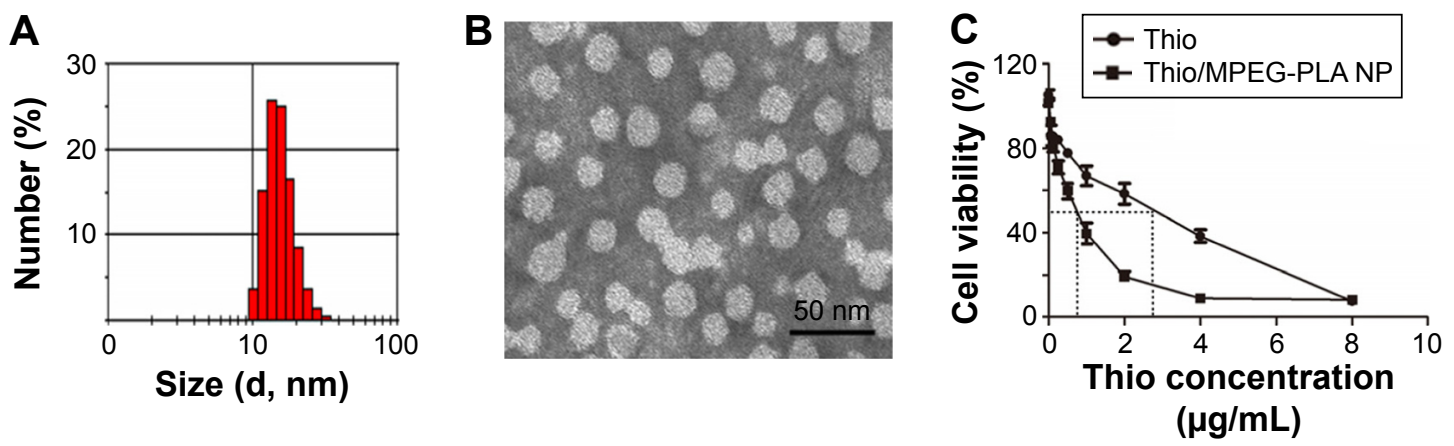

Figure 2 Characterization of Thio/MPEG-PLA NPs.

Notes: (A) Size distribution spectrum of Thio/MPEG-PLA NPs. (B) TEM image of Thio/MPEG-PLA NPs. (C) Cytotoxicity of Thio and Thio/MPEG-PLA NPs. Abbreviations: Thio, thioridazine; MPEG-PLA, methoxy poly(ethylene glycol)-poly(L-lactic acid); NP, nanoparticle; TEM, transmission electron microscopic.

accessible method to improving anticancer effects. ${ }^{26}$ Besides this, MPEG-PLA copolymer-based codelivery of Dox and paclitaxel also showed enhanced anticancer effects with an improved drug delivery system. ${ }^{15}$

In our study, we loaded Thio-Dox into MPEG-PLA NPs by a two-step self-assembly method (Figure 3A). MPEGPLA could self-assemble into micelles and form a coreshell structure in the rehydration process in water due to its amphiphilic property. Evaporation of the Thio and MPEGPLA codissolved solution allowed the two components to be adequately mixed. In this structure, both Thio and Dox were loaded on the NPs, forming a structure. When the in-feed mass ratio of Thio/Dox/MPEG-PLA was $1 / 2 / 40$, the NPs had an EE of $96.30 \%$ (Thio) and $79.16 \%$ (Dox), and DL of $4.71 \%$ (Thio) and $1.98 \%$ (Dox).

The size of Thio-Dox/MPEG-PLA NPs was $27 \mathrm{~nm}$ (Figure 3B), which was much smaller than the dimensional requirement for in vivo application. The transmission electron microscopic image of Thio-Dox/MPEG-PLA NPs is shown in Figure 3C. NP-mediated cellular response was size dependent, and mammalian cells could efficiently uptake NPs with a size $<100 \mathrm{~nm} .^{27,28}$ The Thio-Dox-loaded NPs with a particle size of $\sim 20 \mathrm{~nm}$ were suitable for the drug delivery system. The release profiles of Thio and Dox from the MPEG-PLA NPs were studied using a dialysis method. As shown in Figure 3D and E, both Thio and Dox released slower from the NPs in $\mathrm{pH} 7.4$ than in $\mathrm{pH}$ 5.5. The $\mathrm{pH}$-dependent releasing behavior might be due to the deprotonation of the amino group of Dox and faster degradation of MPEG-PLA NPs at lower $\mathrm{pH} .{ }^{29}$ The drug release of free Thio, free Dox, and Thio-Dox/MPEG-PLA NPs in PBS containing 10\% FBS is shown in Figure 3F and G. The results suggested that free Thio and free Dox released faster than the drug encapsulation by MPEG-PLA NPs. Thus Thio and Dox might be very slowly released in the plasma under normal physiological conditions ( $\mathrm{pH}=7.4$ ) but quickly released at the solid tumor site $(\mathrm{pH}=5.5)$, which was expected to achieve selective release of Thio and Dox in tumor and reduce the systemic toxicity.

\section{Anticancer effects of Thio-Dox-loaded MPEG-PLA NPs in breast cancer in vivo}

The therapeutic effect following the treatment of Dox and Thio formulations in mice-bearing $4 \mathrm{~T} 1$ xenografts is described in Figure 4. Compared with the tumor weight $0.832 \mathrm{~g}$ in NS group, the average weight of tumors in Thiotreated group and Dox-treated group was $0.538 \mathrm{~g}$ and $0.342 \mathrm{~g}$, respectively. The formulation of Dox and Thio showed more significant tumor inhibition effect than Dox or Thio alone, as evidenced by smaller tumor volume and tumor weight (Figure 4A-C).

The anticancer activity of Dox and Thio in breast cancer was further enhanced through the NP-based drug codelivery system. The average weight of Thio-Dox group and ThioDox/MPEG-PLA NPs group was $0.176 \mathrm{~g}$ and $0.122 \mathrm{~g}$, respectively. The tumor inhibitory rates were $35.4 \%$ (free Thio), $58.9 \%$ (free Dox), $78.8 \%$ (free Thio and Dox), and 85.6\% (Thio-Dox/MPEG-PLA NPs) (Figure 4A-C). The increased anticancer effects of Thio-Dox/MPEG-PLA NPs is due to the better drug accumulation in tumor tissues resulting from an EPR effect, ability to overcome multidrug resistance, and better pharmacokinetics profiles in vivo. We further evaluated the effect of Thio and Dox on metastasis of tumor cells. As shown in Figure 4D, free Thio or Dox may inhibit the metastasis of tumor cells, while the combination of Thio and Dox revealed no lung nodules in mice. In addition, we could not observe metastatic lung nodules in mouse treated with Thio and Dox loaded to MPEG-PLA NPs, implying that the drug encapsulation by MPEG-PLA NPs may not have significant effect on breast cancer metastasis or the nonsignificant 
A

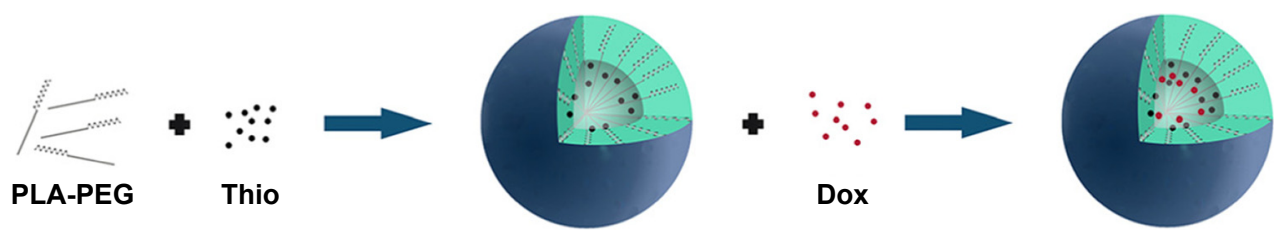

B
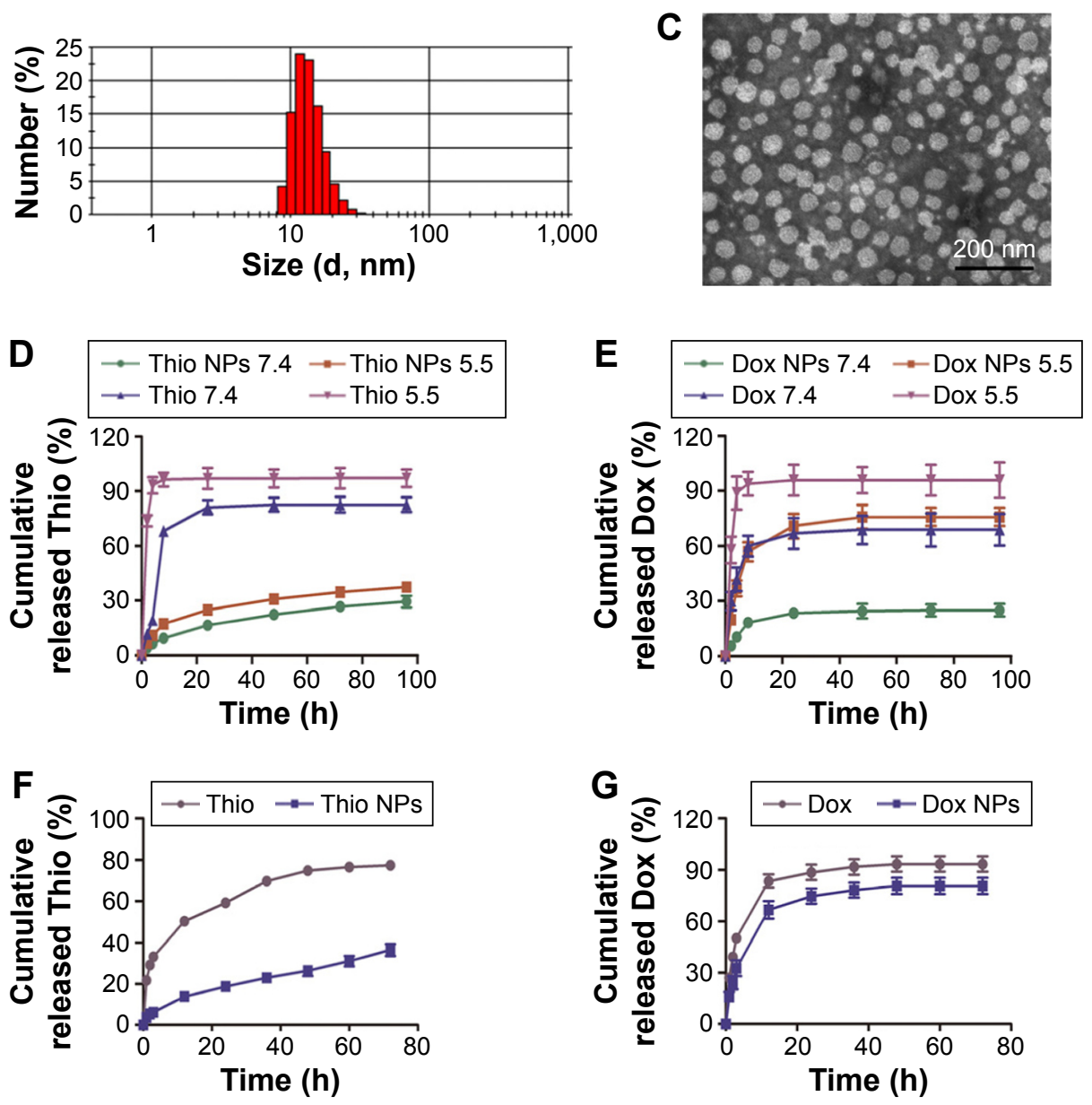

Figure 3 Preparation and characterization of Thio-Dox/MPEG-PLA NPs.

Notes: (A) Synthesis of Thio-Dox/MPEG-PLA NPs. (B) Size distribution spectrum of Thio-Dox/MPEG-PLA NPs. (C) TEM image of Thio-Dox/MPEG-PLA NPs. (D) Drug release of free Thio and Thio/MPEG-PLA NPs in PBS ( $\mathrm{pH} 7.4$ and 5.5). (E) Drug release of free Dox and Dox/MPEG-PLA NPs in PBS (pH 7.4 and 5.5). (F) Drug release of free Thio and Thio/MPEG-PLA NPs in PBS containing $10 \%$ FBS. (G) Drug release of free Dox and Dox/MPEG-PLA NPs in PBS containing I0\% FBS.

Abbreviations: Thio, thioridazine; Dox, doxorubicin; MPEG-PLA, methoxy poly(ethylene glycol)-poly(L-lactic acid); NP, nanoparticle; TEM, transmission electron microscopy; PBS, phosphate-buffered saline; FBS, fetal bovine serum; PLA-PEG, poly(L-lactic acid)-poly(ethylene glycol).

A

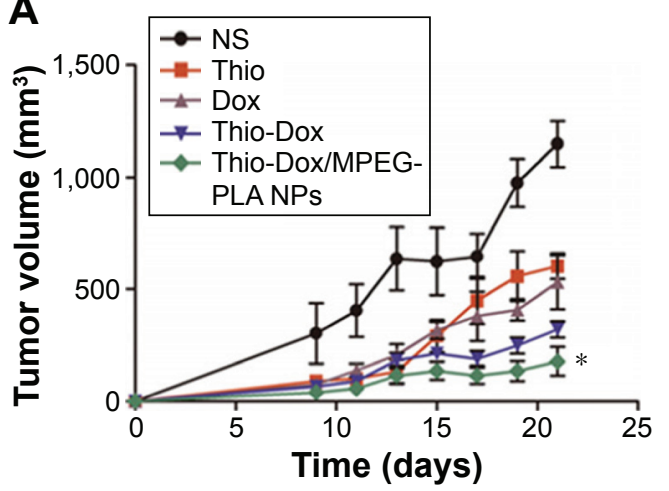

B

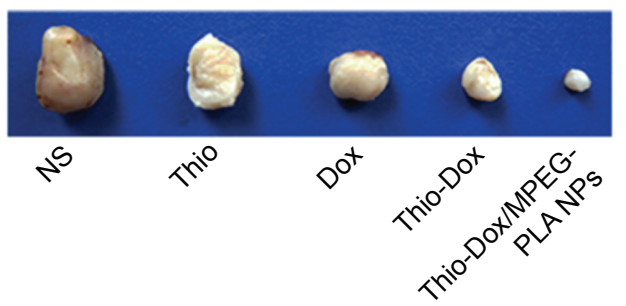

Figure 4 (Continued) 

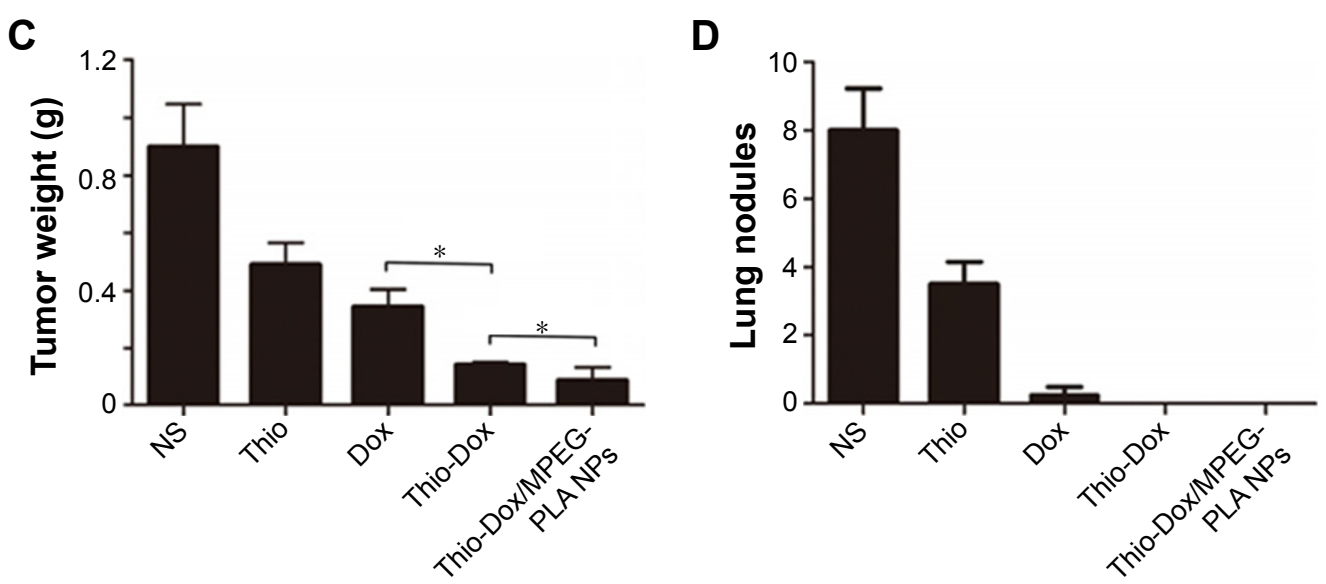

Figure 4 Intravenous injection of Thio-Dox/MPEG-PLA NPs inhibited the growth of breast cancer cells in vivo.

Notes: (A) Tumor development curve. Female mice were subcutaneously transplanted 4TI cells on day 0 . On day 5 , the mice were randomized into five groups and intravenously injected with saline (control), free Thio, free Dox, free Thio and Dox, and Thio-Dox/MPEG-PLA NPs twice a week. (B) Representative photographs of tumors in each treated group on day 22. (C) Tumor weight in different groups on day 22. (D) Numbers of lung nodules in different groups. For all graphs, error bars indicate mean \pm $\mathrm{SD}, * P<0.05$.

Abbreviations: Thio, thioridazine; Dox, doxorubicin; MPEG-PLA, methoxy poly(ethylene glycol)-poly(L-lactic acid); NP, nanoparticle; PEG-PLA, poly(L-lactic acid)poly(ethylene glycol).

effect on metastasis may be due to the marked inhibition of primary tumor growth.

\section{Conclusion}

In this study, Thio and Dox were coencapsulated into biodegradable MPEG-PLA NPs. Compared to free Dox and Thio, the prepared NPs were more efficient in inhibiting the growth of breast cancer cells in vitro and in vivo. Taken together, our study suggests that Thio- and Dox-coloaded MPEG-PLA NPs might have potential application in breast cancer treatment.

\section{Acknowledgments}

This work was supported by the National Natural Science Foundation (81301907, 81422025, and 81572990), Sichuan Provincial Youth Science and Technology Fund (2016JQ0020), and Wu Jieping Medical Foundation (320.6750.12224).

\section{Disclosure}

The authors report no conflicts of interest in this work.

\section{References}

1. Torre LA, Bray F, Siegel RL, Ferlay J, Lortet-Tieulent J, Jemal A. Global cancer statistics, 2012. CA Cancer J Clin. 2015;65(2):87-108.

2. Vargo-Gogola T, Rosen JM. Modelling breast cancer: one size does not fit all. Nat Rev Cancer. 2007;7(9):659-672.

3. Gonzalez-Angulo AM, Morales-Vasquez F, Hortobagyi GN. Overview of resistance to systemic therapy in patients with breast cancer. $A d v$ Exp Med Biol. 2007;608:1-22.

4. Parhi P, Mohanty C, Sahoo SK. Nanotechnology-based combinational drug delivery: an emerging approach for cancer therapy. Drug Discov Today. 2012;17(17-18):1044-1052.
5. Al-Lazikani B, Banerji U, Workman P. Combinatorial drug therapy for cancer in the post-genomic era. Nat Biotechnol. 2012;30(7):679-692.

6. Sachlos E, Risueno RM, Laronde S, et al. Identification of drugs including a dopamine receptor antagonist that selectively target cancer stem cells. Cell. 2012;149(6):1284-1297.

7. Mu J, Xu H, Yang Y, et al. Thioridazine, an antipsychotic drug, elicits potent antitumor effects in gastric cancer. Oncol Rep. 2014;31(5): 2107-2114.

8. Park MS, Dong SM, Kim BR, et al. Thioridazine inhibits angiogenesis and tumor growth by targeting the VEGFR-2/PI3K/mTOR pathway in ovarian cancer xenografts. Oncotarget. 2014;5(13):4929-4934.

9. Tacar O, Sriamornsak P, Dass CR. Doxorubicin: an update on anticancer molecular action, toxicity and novel drug delivery systems. $J$ Pharm Pharmacol. 2013;65(2):157-170.

10. Khayat D, Chollet P, Antoine EC, et al. Phase II study of sequential administration of docetaxel followed by doxorubicin and cyclophosphamide as first-line chemotherapy in metastatic breast cancer. J Clin Oncol. 2001;19(14):3367-3375.

11. Espelin CW, Leonard SC, Geretti E, Wickham TJ, Hendriks BS. Dual HER2 targeting with trastuzumab and liposome-encapsulated doxorubicin (MM-302) demonstrates synergistic antitumor activity in breast and gastric cancer. Cancer Res. 2016;76(6):1517-1527.

12. Amreddy N, Muralidharan R, Babu A, et al. Tumor-targeted and $\mathrm{pH}$-controlled delivery of doxorubicin using gold nanorods for lung cancer therapy. Int J Nanomedicine. 2015;10:6773-6788.

13. Prahalathan C, Selvakumar E, Varalakshmi P. Lipoic acid modulates adriamycin-induced testicular toxicity. Reprod Toxicol. 2006;21(1): $54-59$.

14. Broxterman HJ, Gotink KJ, Verheul HM. Understanding the causes of multidrug resistance in cancer: a comparison of doxorubicin and sunitinib. Drug Resist Updat. 2009;12(4-5):114-126.

15. Lv S, Tang Z, Li M, et al. Co-delivery of doxorubicin and paclitaxel by PEG-polypeptide nanovehicle for the treatment of non-small cell lung cancer. Biomaterials. 2014;35(23):6118-6129.

16. Zhang P, Li J, Ghazwani M, et al. Effective co-delivery of doxorubicin and dasatinib using a PEG-Fmoc nanocarrier for combination cancer chemotherapy. Biomaterials. 2015;67:104-114.

17. Bourzac K. Nanotechnology: carrying drugs. Nature. 2012;491(7425): S58-S60.

18. Bonifacio BV, Silva PB, Ramos MA, Negri KM, Bauab TM, Chorilli M. Nanotechnology-based drug delivery systems and herbal medicines: a review. Int J Nanomedicine. 2014;9:1-15. 
19. Yan L, Yang Y, Zhang W, Chen X. Advanced materials and nanotechnology for drug delivery. Adv Mater. 2014;26(31):5533-5540.

20. Jabr-Milane LS, van Vlerken LE, Yadav S, Amiji MM. Multi-functional nanocarriers to overcome tumor drug resistance. Cancer Treat Rev. 2008; 34(7):592-602.

21. Maeda H, Wu J, Sawa T, Matsumura Y, Hori K. Tumor vascular permeability and the EPR effect in macromolecular therapeutics: a review. $J$ Control Release. 2000;65(1-2):271-284.

22. Iyer AK, Khaled G, Fang J, Maeda H. Exploiting the enhanced permeability and retention effect for tumor targeting. Drug Discov Today. 2006; 11(17-18):812-818

23. Cao N, Feng SS. Doxorubicin conjugated to D-alpha-tocopheryl polyethylene glycol 1000 succinate (TPGS): conjugation chemistry, characterization, in vitro and in vivo evaluation. Biomaterials. 2008; 29(28):3856-3865.

24. Gao X, Wang B, Wei X, et al. Preparation, characterization and application of star-shaped PCL/PEG micelles for the delivery of doxorubicin in the treatment of colon cancer. Int J Nanomedicine. 2013;8:971-982.
25. Upadhyay UM, Tyler B, Patta Y, et al. Intracranial microcapsule chemotherapy delivery for the localized treatment of rodent metastatic breast adenocarcinoma in the brain. Proc Natl Acad Sci U S A. 2014;111(45): 16071-16076.

26. Guo O, Li X, Yang Y, et al. Enhanced 4T1 breast carcinoma anticancer activity by co-delivery of doxorubicin and curcumin with core-shell drugcarrier based on heparin modified poly(L-lactide) grafted polyethylenimine cationic nanoparticles. J Biomed Nanotechnol. 2014;10(2):227-237.

27. Jiang W, Kim BY, Rutka JT, Chan WC. Nanoparticle-mediated cellular response is size-dependent. Nat Nanotechnol. 2008;3(3):145-150.

28. Chithrani BD, Chan WC. Elucidating the mechanism of cellular uptake and removal of protein-coated gold nanoparticles of different sizes and shapes. Nano Lett. 2007;7(6):1542-1550.

29. Shuai X, Ai H, Nasongkla N, Kim S, Gao J. Micellar carriers based on block copolymers of poly(epsilon-caprolactone) and poly(ethylene glycol) for doxorubicin delivery. J Control Release. 2004;98(3):415-426.
International Journal of Nanomedicine

\section{Publish your work in this journal}

The International Journal of Nanomedicine is an international, peerreviewed journal focusing on the application of nanotechnology in diagnostics, therapeutics, and drug delivery systems throughout the biomedical field. This journal is indexed on PubMed Central, MedLine, CAS, SciSearch ${ }^{\circledR}$, Current Contents ${ }^{\circledR} /$ Clinical Medicine,

\section{Dovepress}

Journal Citation Reports/Science Edition, EMBase, Scopus and the Elsevier Bibliographic databases. The manuscript management system is completely online and includes a very quick and fair peer-review system, which is all easy to use. Visit http://www.dovepress.com/ testimonials.php to read real quotes from published authors. 